\title{
Anesthetic management of a patient with Mounier-Kuhn syndrome undergoing off-pump coronary artery bypass graft surgery -A case report-
}

\author{
Jeong Jin Min, Jung-Man Lee, Jun Hyun Kim, Deok Man Hong, Yunseok Jeon, and Jae-Hyon Bahk \\ Department of Anesthesiology and Pain Medicine, Seoul National University Hospital, Seoul, Korea
}

\begin{abstract}
Mounier-Kuhn-syndrome patients have markedly dilated trachea and main bronchi due to an atrophy or absence of elastic fibers and thinning of smooth muscle layers in the tracheobronchial tree. Although this syndrome is rare, airway management is challenging and general anesthesia may produce fatal results. However, only a few cases have been reported and this condition is not widely known among anesthesiologists. We present the case of a tracheobronchomegaly patient undergoing an emergency off-pump coronary artery bypass. Although the trachea was markedly dilated with numerous tracheal diverticuli, there was an undilated $2 \mathrm{~cm}$ portion below the vocal cords found on the preoperative CT. Under a preparation of extracorporeal membrane oxygenation, we intubated and placed the balloon of an endotracheal tube (I.D. $9 \mathrm{~mm}$ ) at this portion, and maintained ventilation during the operation. This case showed that a precise preoperative evaluation and anesthetic plan is essential for successful anesthetic management. (Korean J Anesthesiol 2011; 61: 83-87)
\end{abstract}

Key Words: Mounier-Kuhn syndrome, Tracheal diverticuli, Tracheobronchomegaly.

Patients with Mounier-Kuhn syndrome have markedly dilated trachea and main bronchi due to the atrophy or absence of elastic fibers and thinning of smooth muscle layers in the tracheobronchial tree. Although this syndrome is rare, anesthetic management of this syndrome is challenging and may produce fatal results. If general anesthesia is conducted using a conventional airway management technique, it may be impossible to maintain positive pressure ventilation because of peritubal air leakage or large airway collapse and obstruction. Pulmonary aspiration and tracheal injury could also occur. Thus, preoperative evaluation of a patient's airway and a proper anesthetic plan for airway management are essential. However, only a few cases of Mounier-Kuhn patient anesthetic management have been reported and this syndrome is not

Received: December 1, 2010. Revised: February 8, 2011. Accepted: February 9, 2011.

Corresponding author: Yunseok Jeon, M.D., Ph.D., Department of Anesthesiology and Pain Medicine, Seoul National University Hospital, Yeongun-dong, Jongro-gu, Seoul 110-744, Korea. Tel: 82-2-2072-2465, Fax: 82-2-747-5639, E-mail: jeonyunseok@gmail.com (ㄷ) This is an open-access article distributed under the terms of the Creative Commons Attribution Non-Commercial License (http:// creativecommons.org/licenses/by-nc/3.0/), which permits unrestricted non-commercial use, distribution, and reproduction in any medium, provided the original work is properly cited. 
widely known among anesthesiologist. We experienced a 62-year-old man diagnosed with Mounier-Kuhn syndrome during preoperative evaluation for an emergency off-pump coronary artery bypass surgery (OPCAB). We report this case with a review of the relevant literature.

\section{Case Report}

A 62-year-old, $56.7 \mathrm{~kg}, 160.4 \mathrm{~cm}$ man was scheduled for an emergency OPCAB surgery. The patient has medical history of hypertension and diabetes mellitus with insulin therapy. He had also been recently diagnosed with unstable angina and three vessel coronary arterial disease with significant left main coronary arterial stenosis. In the preoperative review of the chest computed tomography (CT) angiography, a dilated trachea and main bronchi with numerous tracheal diverticuli (Fig. 1) were observed. On the chest CT, the maximum tracheal diameter was $26.31 \mathrm{~mm}$ (transverse) and $35.86 \mathrm{~mm}$ (sagittal plane), and Mounier-Kuhn syndrome was diagnosed (Fig. 2). The patient was a non-smoker and his preoperative respiratory function tests and arterial blood gas analysis were normal $(\mathrm{pH}$ 7.37; $\mathrm{PaCO}_{2}, 45 \mathrm{mmHg} ; \mathrm{PaO}_{2}, 96 \mathrm{mmHg} ; \mathrm{HCO}_{3}{ }^{-}, 26 \mathrm{mEq} / \mathrm{L}$;
$\mathrm{SaO}_{2}, 97 \%$ in air). The preoperative bronchoscopic exam found tracheal expansions with diverticulosis and no endobronchial lesions. After a review of the chest CT, we found an undilated portion of the trachea below vocal cords approximately 2

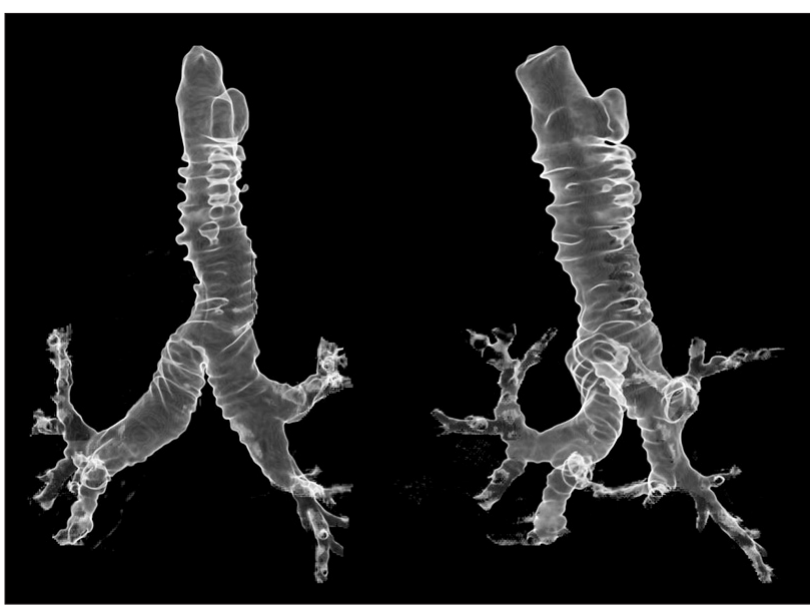

Fig. 1. Preoperative chest CT (3D reconstruction) showed markedly dilated trachea and main bronchi with numerous tracheal diverticuli. Anteroposterior (left) and lateral (right) views.
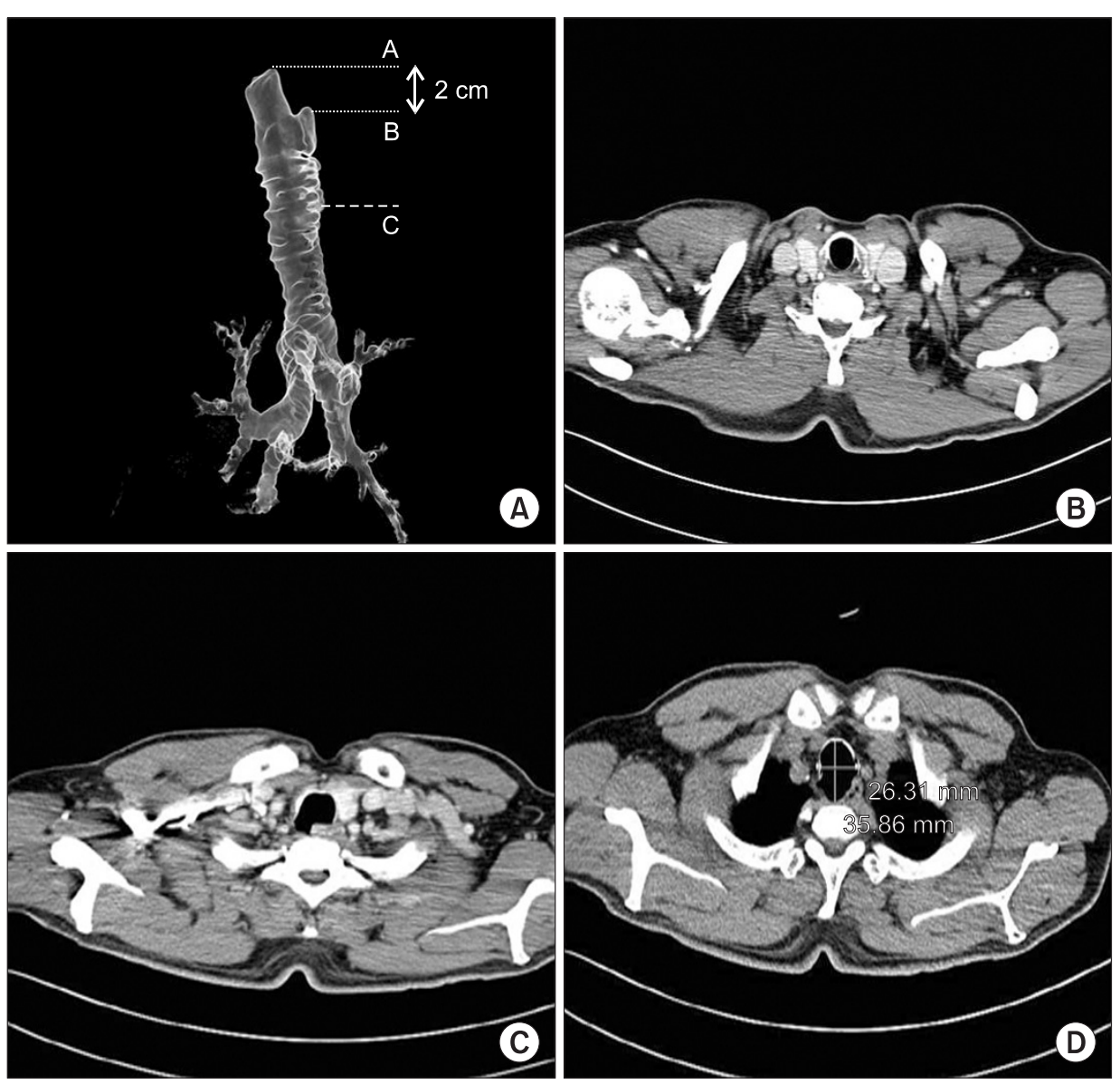

Fig. 2. (A) Lateral view of the tracheobroncheal tree on 3D chest CT angiogram. There was a relatively undilated portion of the trachea just below the vocal cords (from point A to B). (B) Axial plane of the chest CT scan showing relatively normal sized trachea (at point A in Fig. 2A). (C) Axial plane of the chest CT scan with a diverticulum (at point B in Fig. 2A). (D) Axial plane of the chest CT scan showing the maximum trachea diameter (at point $\mathrm{C}$ in Fig. 2A). 
cm long (Fig. 2) and planned to place the balloon of the endotracheal tube in this area. In the operating room, we also prepared endotracheal tubes of various sizes, laryngeal mask airway (LMA), fiberoptic bronchoscopy, and extracorporeal membrane oxygenation (ECMO) with cardiac surgeons before anesthesia.

The patient arrived to the operating room with an intraaortic balloon pump and continuous intravenous nitroglycerin administration ( $3 \mu \mathrm{g} / \mathrm{kg} / \mathrm{min}$ ). Before anesthesia, arterial blood pressure monitoring was started with $1 \mathrm{mg}$ of intravenous midazolam. After sufficient preoxygenation with $100 \%$ oxygen, anesthesia was induced with $20 \mathrm{mg}$ etomidate and $100 \mathrm{mg}$ succinylcholine injected intravenously. After successful manual mask ventilation, we intubated the patient with an internal diameter (I.D.) $9 \mathrm{~mm}$ cuffed endotracheal tube to place balloon just below the vocal cords (Fig. 3). After fixing the tube $18 \mathrm{~cm}$ from the patient's incisor, we checked the trachea using fiberoptic bronchoscopy and confirmed that the lumen of the trachea was relatively preserved. Anesthesia was maintained with a continuous intravenous infusion of remifentanil, propofol, and vecuronium, and patient's lung was ventilated with a volume controlled mode with $50 \%$ oxygen. A nasogastric Levin tube was inserted for gastric decompression and prevention of pulmonary aspiration. There was intermittent air leakage around the endotracheal tube accompanied with the patient's procedural position changes but it was successfully managed by slightly adjusting the depth of the endotracheal

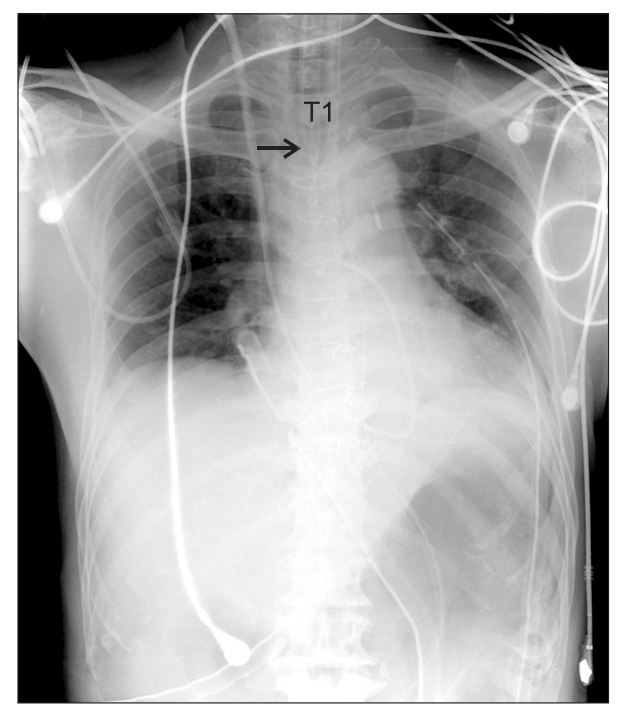

Fig. 3. Postoperative anteroposterior chest x-ray. A Black arrow indicates the distal tip of the endotracheal tube. The distal tip of the endotracheal tube was below the first thoracic vertebra (T1) level. Considering that the level of the larynx is at sixth cervical vertebra (C6) level in adults, the balloon of the endotracheal tube might be located just below the vocal cords. tube. The OPCAB was successfully completed in 6 hours. During the procedure, the patient's airway and ventilation were managed without further manipulations. In the postoperative intensive care unit, the patient was managed by the same strategy as in the operating room, and extubated the next day. After 5 days, he was discharged from the hospital without any respiratory complications.

\section{Discussion}

Mounier-Kuhn syndrome is a rare disorder characterized by marked dilatation of the trachea and main bronchi, and is sometimes associated with tracheal diverticulosis or bronchiectasis $[1,2]$. The exact prevalence of this disease is not known and fewer than a hundred cases have been reported. Anesthetic experience with these patients is very limited and only a few cases have been reported to date. This syndrome is slightly more common in males usually in their 30 s or 40 s although some cases have been reported in patients 18 months to 81 years old $[3,4]$.

For an adult, any diameter of the trachea, right main bronchus, and left main bronchus that exceeds $3.0 \mathrm{~cm}, 2.4 \mathrm{~cm}$, and $2.3 \mathrm{~cm}$, respectively, on a chest radiograph is indicative of Mounier-Kuhn syndrome because these values are upper limits of the means plus three standard deviations $[5,6]$. On chest CT, this syndrome is diagnosed in men when the transverse and the sagittal diameters of the trachea exceed $25 \mathrm{~mm}$ and $27 \mathrm{~mm}$, respectively, and when the transverse diameters of the right and left main bronchi exceed $21.1 \mathrm{~mm}$ and $18.4 \mathrm{~mm}$, respectively. In women, the transverse and sagittal diameters of the trachea exceed $21 \mathrm{~mm}$ and $23 \mathrm{~mm}$, respectively, and the transverse diameters of the right and left main bronchi exceed $19.8 \mathrm{~mm}$ and $17.4 \mathrm{~mm}$ [7] for this diagnosis. In our 62-year-old male case, the maximum transverse and sagittal diameters of the trachea were $26.31 \mathrm{~mm}$ and $35.86 \mathrm{~mm}$, and the transverse diameters of the right and left main bronchi were $24.75 \mathrm{~mm}$ and $22.34 \mathrm{~mm}$, indicating Mounier-Kuhn syndrome.

The etiology of this syndrome is uncertain. Autopsy studies have suggested a congenital defect or atrophy of the elastic and smooth muscle tissues of the trachea and main bronchi, and a familial form has been reported with a possible recessive inheritance $[6,8]$. Mounier-Kuhn syndrome also may be associated with some rare genetic disorders including Brachmann-de Lange syndrome and connective tissue diseases such as rheumatoid arthritis, Ehlers-Danlos, and Cutis Laxa. However, acquired forms of tracheobronchomegaly have also been described complications of pulmonary fibrosis in adults and mechanical ventilation in preterm neonates. Furthermore, during prolonged mechanical ventilation with endotracheal intubation, acquired tracheomalacia or tracheomegaly may 
result from pressure and impairment of blood supply in the tracheal wall, infection, and cyclic friction on dry tracheal mucosa [9].

Although the patient in our case had no history of recurrent airway infections, the usual clinical presentation of MounierKuhn syndrome includes recurrent lower respiratory tract infections with a broad spectrum of functional impairments ranging from minimal disease with good preservation of pulmonary function to severe forms leading to respiratory failure and death. Grossly enlarged but weakened airways and inefficient coughing block mucociliary clearance leading to mucous retention with subsequent recurrent pneumonia, bronchiectasis, and fibrosis. Excessive sputum production with occasional hemoptysis occurs and patients may develop dyspnea and respiratory failure as the lungs become progressively damaged [10]. Pulmonary function tests may reveal an obstructive pattern but could show various patterns according to the disease state and in advanced cases with pulmonary fibrosis, restrictive patterns may be predominant. In our patient, the preoperative respiratory function tests were normal.

For anesthesiologists, the major concern with the patients of Mounier-Kuhn syndrome is thus airway management. Due to atrophy or absence of elastic fibers and thinning of smooth muscle layers in the trachea and main bronchi, airways in these patients are flaccid and susceptible to marked dilatation during inspiration and airway collapse or obstruction upon expiration. There may be increased dead space and diminished secretion clearing. Above all, due to the abnormally dilated trachea, anesthesiologists may encounter difficulties in maintaining positive pressure ventilation using usual sizes of cuffed endotracheal tubes, and severe peritubal air leakage can increase the risk of hypoventilation or pulmonary aspiration.

In our case, in preanesthetic chest CT review, we found a relatively undilated portion of trachea at just below the vocal cords approximately $2 \mathrm{~cm}$ in length and placed the cuff within this area using an endotracheal tube with a larger internal diameter than usual. In case of failure to provide adequate ventilation, we also prepared variable sizes of endotracheal tubes, LMA, fiberoptic bronchoscopy, and ECMO on dry conditions before anesthesia. Fortunately, we succeeded in ventilating the patient using an I.D $9.0 \mathrm{~mm}$ cuffed endotracheal tube as originally planned.

In a previous anesthetic experience reported by Kim et al. [4], the patient was scheduled to undergo an elective tracheostomy in Korea. However, because the regular tracheostomy tube was not long enough to allow the cuff to lie properly in the trachea, the tracheostomy could not be performed. Due to inadequate ventilation resulting from peritracheostomy tubal air leakage, the patient's arterial blood gas showed respiratory acidosis and hypercapnia (Ph 7.09; $\mathrm{PaCO}_{2}, 97.2$ mmHg; $\mathrm{PaO}_{2}, 78$ mmHg; and $\left.\mathrm{HCO}_{3}{ }^{-}, 29.7 \mathrm{mEq} / \mathrm{L}\right)$. In our case, the preanesthetic chest CT review helped to determine the airway management plan.

In addition, tracheal injury may follow endotracheal tube intubation due to the weakened airways. Although the patient in our case was extubated and discharged from hospital without respiratory complications, there is one previous report of tracheal stenosis developing at the site of previous dilatation in a tracheobronchomegaly patient who was intubated for 15 days with an endotracheal tube with a high-volume, lowpressure cuff [11]. The resulting stenosis was so severe that the patient underwent resection-anastomosis surgery of the stenotic tracheal segment 2 months after extubation [11]. After this experience, using an uncuffed tube with throat packing was recommended for Mounier-Kuhn syndrome patients, but this isn't thought to be sufficiently safe for preventing hypoventilation or aspiration pneumonitis.

To avoid respiratory complications like tracheal trauma following endotracheal instrumentation, Yasuhiko et al. [12] used the ProSeal LMA instead of usual endotracheal tube intubation in a Mounier-Kuhn syndrome patient who underwent ovarian cancer operation. The 7-hour operation was uneventful and the patient awoke without any respiratory complications. For procedures with short durations and low chances of LMA displacement, airway management with LMA could be a good way to avoid tracheal injury. However, for operations with long durations or possible position changes during the procedure, LMA displacement could occur, leading to inadequate patient ventilation. Furthermore, maintaining positive pressure ventilation using LMA can be limited in patients with decreased lung compliance, increased airway resistance, increased risk of pulmonary aspiration, and in some procedures that need one lung ventilation. In conclusion, although patients with Mounier-Kuhn syndrome could have only few non-specific preoperatively symptoms, general anesthesia could be fatal without appropriate management. Therefore, precise preoperative evaluation, an anesthetic plan, and preparation are essential for successful anesthetic management of Mounier-Kuhn syndrome patients.

\section{References}

1. Bateson EM, Woo-Ming M. Tracheobronchomegaly. Clin Radiol 1973; 24: 354-8.

2. Gay S, Dee P. Tracheobronchomegaly--the Mounier-Kuhn syndrome. Br J Radiol 1984; 57: 640-4.

3. Hunter TB, Kuhns LR, Roloff MA, Holt JF. Tracheobronchomegaly in an 18 month old child. Am J Roentgenol Radium Ther Nucl Med 1975; 123: 687-90.

4. Kim MY, Kim EJ, Min BW, Ban JS, Lee SK, Lee JH. Anesthetic experience of a patient with tracheomegaly: A case report. Korean J 
Anesthesiol 2010; 58: 197-201.

5. Breatnach E, Abbott GC, Fraser RG. Dimensions of the normal human trachea. AJR Am J Roentgenol 1984; 142: 903-6.

6. KATZ I, LEVINE M, HERMAN P. Tracheobronchiomegaly. The Mounier-Kuhn syndrome. Am J Roentgenol Radium Ther Nucl Med 1962; 88: 1084-94.

7. Woodring JH, Howard RS 2nd, Rehm SR. Congenital tracheobronchomegaly (Mounier-Kuhn syndrome): a report of 10 cases and review of the literature. J Thorac Imaging 1991; 6: 1-10.

8. JOHNSTON RF, GREEN RA. Tracheobronchomegaly. Report of five cases and demonstration of familial occurrence. Am Rev Respir Dis
1965; 91: 35-50.

9. Feist JH, Johnson TH, Wilson RJ. Acquired tracheomalacia: etiology and differential diagnosis. Chest 1975; 68: 340-5.

10. Van Schoor J, Joos G, Pauwels R. Tracheobronchomegaly--the Mounier-Kuhn syndrome: report of two cases and review of the literature. Eur Respir J 1991; 4: 1303-6.

11. Messahel FM. Tracheal dilatation followed by stenosis in MounierKuhn syndrome. A case report. Anaesthesia 1989; 44: 227-9.

12. Imashuku Y, Kitagawa H, Fukushima Y, Aoi R. Anesthesia with the ProSeal Laryngeal Mask Airway for a patient with Mounier-Kuhn syndrome. J Clin Anesth 2010; 22: 154. 